\title{
TRACIONAMENTO DE CANINO MANDIBULAR IMPACTADO APÓS CIRURGIA DE ODONTOMA PARA POSTERIOR CORREÇÃO DA MALOCLUSÃO: RELATO DE CASO
}

\author{
Impacted Mandibular Canine Traction After Odontoma Surgery For Subsequent Malocclusion \\ Correction: Case Report
}

Letícya Rezende de Oliveira ${ }^{1}$, Rafaella de Paiva Floriano ${ }^{1}$, Nathália Vieira Duarte른, Winícius Arildo Ferreira Araújo Marcelo Costa Rodrigues ${ }^{4}$, Grace Kelly Martins Carneiro ${ }^{5 *}$

\begin{abstract}
RESUMO
Muitos caninos retidos possuem um folículo pericoronário bem desenvolvidos, que radiograficamente são vistos como uma área radiolúcida ao redor da coroa dental. Acredita-se que tal folículo pode gerar cistos ou tumores odontogênicos. Quando a erupção livre não acontece e o dente não erupciona após exposição cirúrgica, é necessário o auxílio do tracionamento ortodôntico para o seu correto posicionamento na arcada. O objetivo deste estudo foi relatar um caso clínico de tracionamento ortodôntico de um canino inferior incluso devido a presença de um odontoma composto, para posteriormente realizar a correção da maloclusão. O presente trabalho apresenta um caso clínico de uma paciente do sexo feminino de 11 anos de idade, diagnosticada com odontoma que impedia a erupção do canino inferior esquerdo impactado. Foi realizado o acesso cirúrgico para a remoção do odontoma, seguido de colagem de ganchos para o tracionamento ortodôntico ancorado a uma placa rígida. Após o sucesso no tracionamento do dente impactado, foi planejado o aparelho torno invertido para a correção da mordida cruzada anterior, realizando ativações de 1/4 volta a cada 20 dias até o completo descruzamento da arcada. A utilização de aparelhos ortodônticos tanto para tração de dentes impactados como para a correção da maloclusão, apresentam muitos relatos de sucesso na literatura, desde que diagnosticados e tratados precocemente para não agravar o prognóstico do tratamento.

Palavras-chave: Dente Impactado; Diagnóstico; Odontoma; Maloclusão;
\end{abstract}

\section{ABSTRACT}

Many retained canines have a well-developed pericoronal follicle, which radiographically is seen as a radiolucent area around the dental crown. It is believed that such a follicle can generate odontogenic cysts or tumors. When free eruption does not occur and the tooth does not erupt after surgical exposure, the aid of orthodontic traction is necessary for its correct positioning in the arch. The aim of this study was to report a clinical case of orthodontic traction of an impacted mandibular canine due to the presence of a compound odontoma, to perform the malocclusion correction. This paper presents a clinical case of an 11-year-old female patient diagnosed with odontoma that prevented the eruption of the impacted lower left canine. Surgical access was performed to remove the odontoma, followed by gluing hooks for orthodontic traction anchored to a rigid plate. After the successful traction of the impacted tooth, the inverted lathe appliance was planned to correct the anterior crossbite, performing $1 / 4$ turn activations every 20 days until the arch was completely uncrossed. The use of orthodontic appliances both for traction of impacted teeth and for the correction of malocclusion has many successful reports in the literature, provided they are diagnosed and treated early so as not to worsen the treatment prognosis.

Keywords: Impacted Tooth; Diagnosis; Odontoma; Malocclusion.

1. Acadêmica do curso de Odontologia da Faculdade Morgana Potrich (FAMP), Mineiros - GO, Brasil.

2. Cirurgiã Dentista - Curso de Odontologia da Faculdade Morgana Potrich (FAMP), Mineiros - GO, Brasil.

3. Aluno de Pós-graduação em Odontologia, área de concentração em Estomatologia da Universidade Estadual Paulista Júlio de Mesquita filho/ UNESP.

4. Mestre em Biociência Animal, área de concentração em Nutrição Animal/UFJ.

5. Mestre em Ortodontia pela São Leopoldo Mandic / SP, Especialista em Ortodontia pela EAP / Goiás, Especialista em Endodontia pela APCD / SP. Docente do curso de Odontologia da Faculdade Morgana Potrich Eireli - FAMP, Mineiros - GO, Brasil.

*Autor para Correspondência. E-mail: gracekelly@fampfaculdade.com.br 


\section{INTRODUÇÃO}

Define-se impactação dentária, o dente que, chegada a época cronológica de erupção, permanece parcial ou totalmente contido dentro do osso alveolar, com preservação ou não do folículo pericoronário. Os dentes, quando mantidos impactados, podem acarretar uma série de transtornos ao paciente, tais como: pericoronarite, reabsorção radicular do dente adjacente e/ou formação de uma lesão cística ${ }^{(1-5)}$.

Um dos dentes com prevalência de impactação é o canino, o qual é um elemento dental com uma grande importância para se conseguir uma oclusão balanceada e uma boa estética, contudo existe uma prevalência de impactação, podendo causar consequências tanto na estética quanto na fonética do paciente ${ }^{(5)}$. A ocorrência de caninos impactados tem predileção pelo sexo feminino e pela região da maxila $(6,7)$.

Quando ocorre a ausência de elemento dentário, fazse necessário um exame clínico e radiográfico detalhado, sendo a principal forma de diagnóstico, a fim de verificar a causa. Na maioria das vezes a impactação dentária pode estar associada a algum tipo de lesão, como o odontoma que são tumores odontogênico benignos que se manifestam como lesões assintomáticas de crescimento lento e na maioria das vezes o sinal clínico é a ausência do dente que se encontra retido. Sua classificação consiste em dois tipos de odontoma, o composto que possui como principal característica a presença de vários dentículos em seu interior, sendo evidenciado por uma imagem radiopaca de diversos dentículos de tamanhos diferentes, circunscrito por um halo radiolúcido. Já o complexo é marcado por uma massa tecidual ${ }^{(8)}$, apresenta-se como uma massa radiopaca amorfa envolvida também por uma margem radiolúcida. O diagnóstico e tratamento precoce associados aumentam as chances de preservação desse dente. Geralmente o tratamento consiste na enucleação simples seguida de curetagem, posteriormente quando necessário a tração do dente impactado $^{(9)}$.

Dentre as formas de tratamento, o tracionamento do dente incluso deve ser considerado. Várias formas são descritas na literatura para tracionar e alinhar caninos inclusos, dentre elas os aparelhos ortodônticos fixos ou removíveis, a aplicação de ancoragem na mesma arcada ou na arcada oposta e a aplicação de forças vindas de magnetos associados à placa removível. Em alguns casos a aplicação de mini implantes é essencial para a ancoragem dos dentes retidos. Gancho, botão ou tela ligada a correntinha, são mecanismo que facilitam o tratamento, sendo a ativação, realizada por meio da diminuição do comprimento da corrente $^{(10)}$.
Quando a impactação dental está associada a mordida cruzada anterior, deve-se ressaltar que a alteração na oclusão não se autocorrige ao longo do tempo, pelo contrário, tende a ser agravada com o crescimento e desenvolvimento, dificultando e agravando o prognóstico do tratamento. Desta forma, deve ser diagnosticada e tratada o mais precocemente possível ${ }^{(11)}$. Desse modo, este trabalho tem como objetivo apresentar um relato de caso de tracionamento ortodôntico de um canino inferior incluso devido à presença de um odontoma composto, e realizar a correção da maloclusão.

\section{RELATO DE CASO CLÍNICO}

Paciente do sexo feminino, aos 11 anos de idade, procurou a Clínica da Faculdade FAMP, para exame de rotina. Ao exame clínico foi observado ausência do canino inferior esquerdo permanente (33) assim como agenesia dos dentes 12 e 22 e mordida cruzada anterior. Foi realizada uma radiografia panorâmica a qual apresentava uma massa radiolúcida com focos radiopacos envolvendo a coroa do dente (Figura 1).

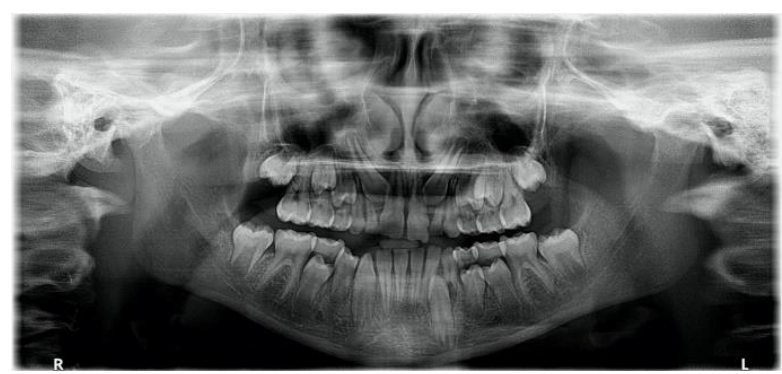

Figura 1 - Radiografia panorâmica. Fonte: Raio x Oral

Para melhor avaliação, foi solicitada uma tomografia computadorizada (Figura 2) a qual comprovou a presença de uma lesão mista. Como hipóteses diagnósticas foram sugeridos odontoma e cisto odontogênico calcificante.

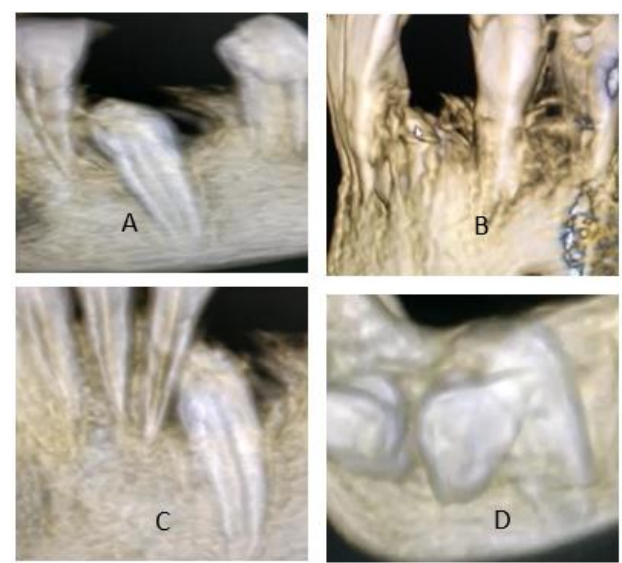

Figura 2 - Tomografia computadorizada. A- Presença do halo radiolúcido. B- Odontoma por vestibular dentro do capuz. C- Canino na posição vertical. D- Inclinação do canino por palatino. Fonte: Raio x Oral 
Após a autorização do paciente, realizou-se o protocolo fotográfico, com fotos extrabucais e intraorais (Figura 3) e modelo de estudo, realizado através da moldagem com alginato. Em seguida com a análise das fotografias foi proposto o plano de tratamento, em que consistiu na remoção cirúrgica do tumor odontogênico, tracionamento do canino e correção da mordida cruzada anterior.
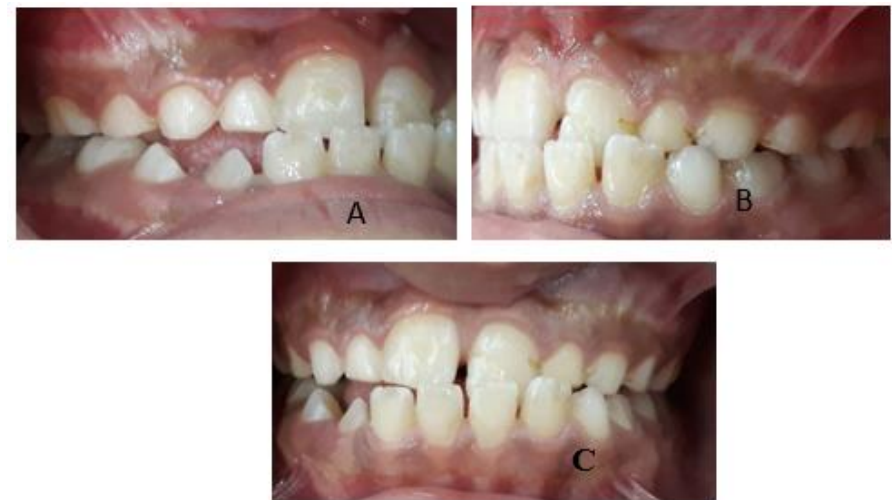

Figura 3 - Fotos intrabucais: A- Intrabucal lateral direita. B- Intrabucal lateral esquerdo. C- Intrabucal Frontal.

Antes de realizar o processo cirúrgico de remoção do tumor odontogênico, optou-se pela moldagem de trabalho para confecção de uma placa de acrílico removível permitindo uma ancoragem satisfatória para tracionamento do canino impactado (Figura 4). Após a prova da placa na boca da paciente, foram realizados os ajustes oclusais (Figura $5)$.
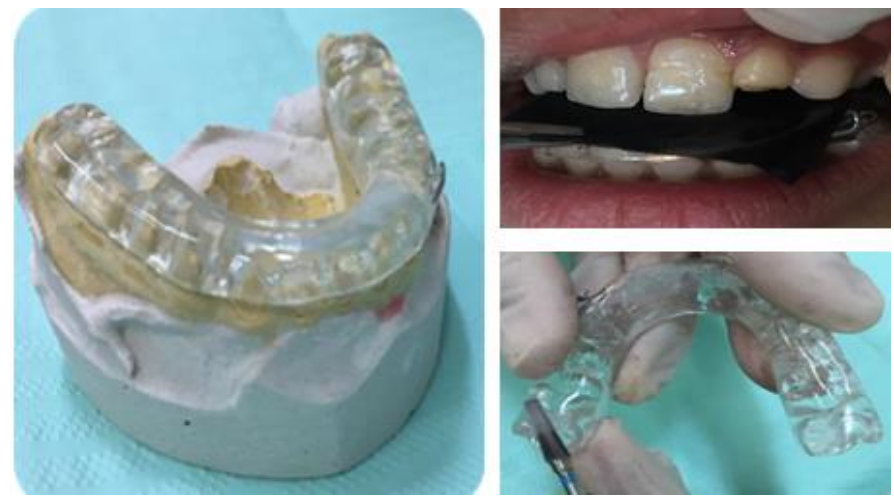

Figura 4 e 5 - Placa de acrílico com gancho para tracionamento de canino impactado. Ajustes na placa rígida. Fonte: Arquivo pessoal

Iniciou a cirurgia de acesso sob anestesia local, com a técnica do bloqueio mandibular (Figura 6) utilizando como anestésico de escolha, lidocaína 2\% 1:1000.000 com vasoconstritor e agulha longa.

Prosseguiu com uma relaxante na região do incisivo lateral (Figura 7), descolamento do tecido gengival com descolador de Molt 2-4 (Figura 8) e remoção da tábua óssea

que circundava a coroa dentária com broca cirúrgica 702 (Figura 9). Foi realizada a remoção do tumor odontogênico (Figura 10) e exposição da coroa do canino impactado (Figura 11). Utilizou-se o ataque ácido com ácido fosfórico a $37 \%$ (Figura 12) e a colagem do gancho ortodôntico com resina Orthocem no dente 33 (Figura 13) a fim de aproveitar a loja cirúrgica aberta para a realização do tracionamento do canino impactado. Por fim, realização da sutura com fio de nylon 4.0 (Figura 14) e utilização de cimento cirúrgico para recobrimento da área cirúrgica exposta para proporcionar melhor cicatrização e conforto no pós-operatório. O material foi coletado e enviado para análise em laboratório de anatomia patológica da Universidade de São Paulo - USP, confirmando o diagnóstico de Odontoma.

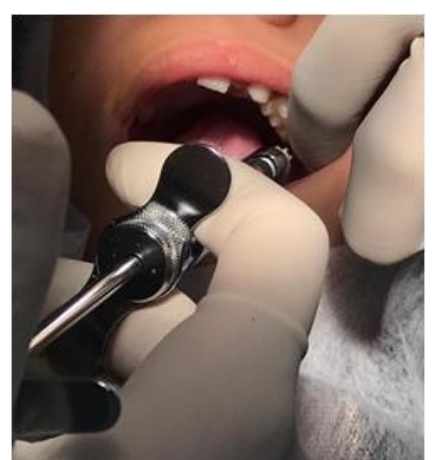

Figura 6 - Anestesia local com a técnica do bloqueio mandibular.

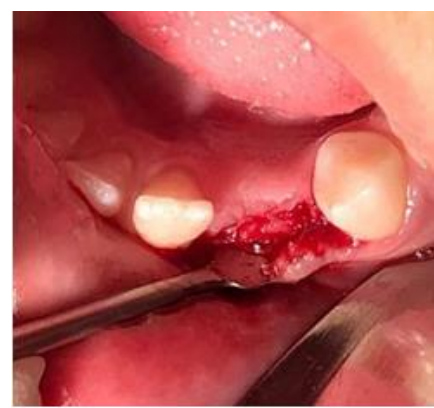

Figura 8- Descolamento do tecido gengival

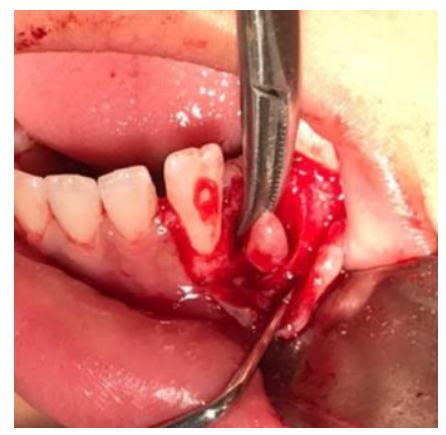

Figura 10 - Remoção do tumor odontogênico

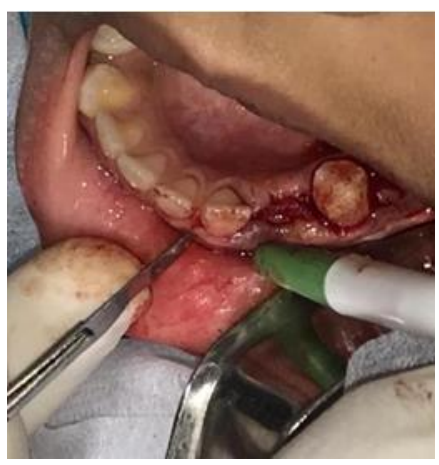

Figura 7 - Relaxante

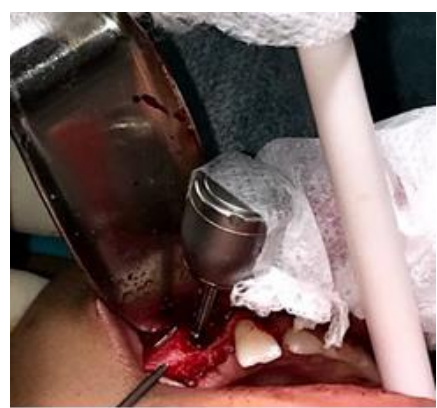

Figura 9 - Remoção da tábua óssea

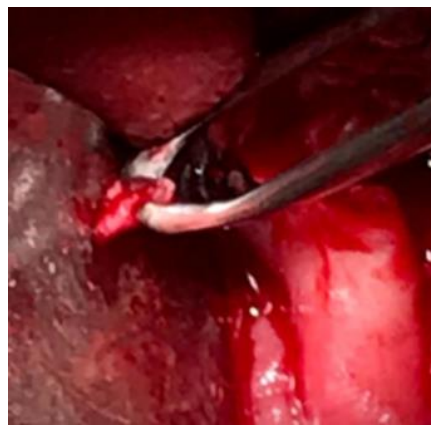




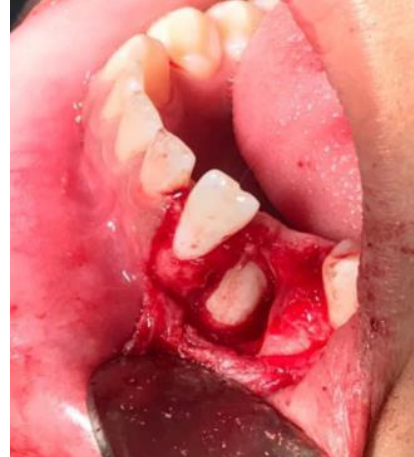

Figura 11- Exposição da coroa do canino impactado

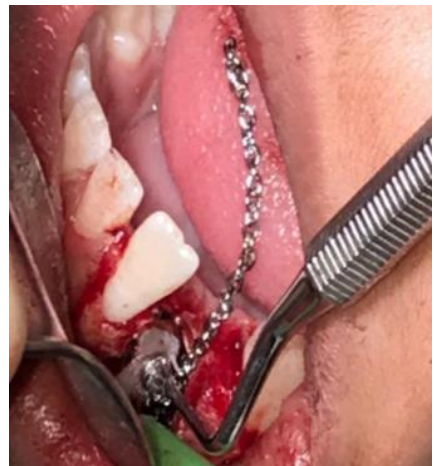

Figura 13- Colagem do gancho Ortodôntico

Todos os cuidados necessários no pós-operatório para uma melhor recuperação foram passados para a criança e seu responsável. Após 7 dias (tempo de cicatrização) removeu cimento cirúrgico e a sutura, observando uma cicatrização satisfatória e iniciou no mesmo dia o tracionamento, ativando a cada 20 dias o fio de amarrilho ortodôntico de 0,25mm (Fig. 15). Após 60 dias, a coroa clínica do elemento 33 rompeu na arcada (Fig. 16) e a menor foi orientada em continuar usando o aparelho para proporcionar maior exposição da coroa clínica. Após erupção do elemento 33, iniciou-se a confecção do torno invertido superior (Fig. 17) aparelho para descruzar a mordida na arcada superior, realizando as ativações a cada 20 dias, ativando $1 / 4$ volta, até ocorrer o descruzamento da maxila. A paciente e o responsável foram orientados quanto ao uso dos aparelhos e higienização. A família da paciente precisou mudar de estado devido as condições de trabalho, foi realizado um encaminhado para um profissional a fim de dar continuidade no caso clínico.

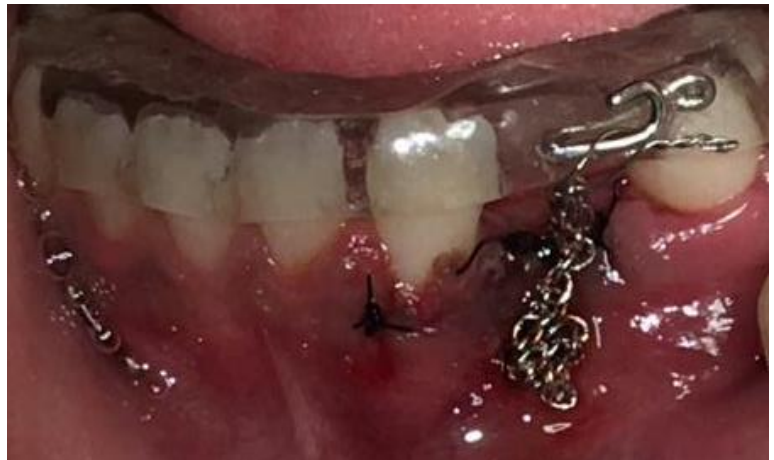

Figura 15- Ativação utilizando fio de amarrilho 0,25.

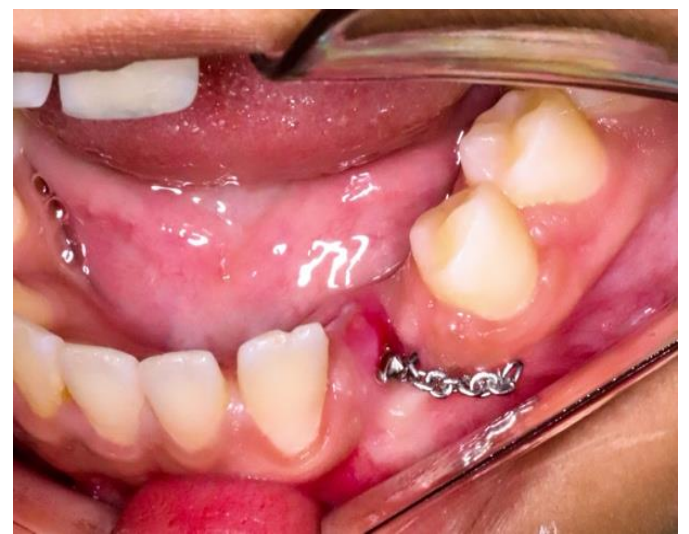

Figura 16 - Erupção do canino impactado após tracionamento

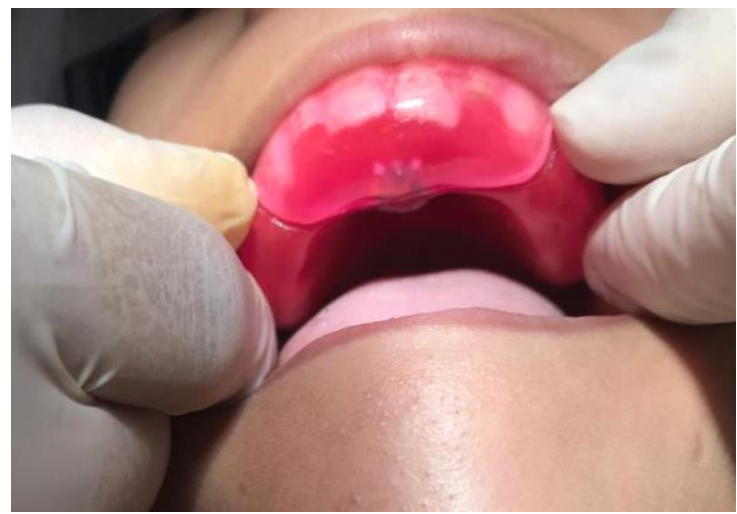

Figura 17- Instalação do torno invertido superior

\section{DISCUSSÃO}

Os caninos desempenham uma importante função no meio bucal, tanto na estética, quanto no aspecto funcional, sendo indispensáveis para a mastigação e essenciais para a oclusão. Quando esse elemento dentário não consegue achar a posição correta no arco dentário, há o aumento de chances de impactação dentária ${ }^{(12)}$.

A impactação do canino é mais comum em pessoas do sexo feminino tendo uma incidência de $2 \%$ em comparação com pessoas do sexo masculino e ocorrem maior número de casos de impactação na maxila, os valores refletem o dobro em comparação com a mandíbula. A 
porcentagem em que ocorrem casos de impactação bilateral é de $8 \%$ na população em geral ${ }^{(13)}$. Já na amostra de Silva ${ }^{(14)}$ (2019) constituída por 40 crianças, 52,5\% do gênero feminino e $47,5 \%$ do gênero masculino, observou-se a erupção de 61,3\% impactação bilateral e a impactação unilateral de 3,8\% dos caninos, os restantes apenas alteraram de posição. Aproximadamente 1/3 dos caninos maxilares impactados estão localizados por vestibular, enquanto $2 / 3$ estão localizados por palatino. Em outros estudos, os autores encontraram uma prevalência de impactação na mandíbula em torno de $0,1 \%$ a $0,31 \%{ }^{(15)}$.

Os tratamentos de dentes impactados podem ser classificados como: tratamentos conservadores não cirúrgicos, (que visam à manutenção do elemento dentário sem qualquer abordagem) a não conservadores (que consistem na eliminação por meio de exodontia) e as conservadoras cirúrgicas (que consistem na manutenção do dente retido, mas com exposição a traumas cirúrgicos para tracionamento ortodôntico). Caso, após avaliações clínicas e radiográficas, não seja indicado tracionamento ortodôntico e a manutenção do dente incluso possa promover danos maiores, a sua remoção é a alternativa indicada ${ }^{(16)}$.

Se o elemento dental estiver em posição de erupção favorável (Face incisal da coroa voltada para o rebordo alveolar) e com o ápice radicular aberto, haverá grandes chances de erupção espontânea, mesmo diante de uma dilaceração radicular ${ }^{(17)}$. Já outros autores relataram que o canino impactado localizado por vestibular está associado mais frequentemente à falta de espaço no arco dentário (83\%), enquanto o canino impactado localizado por lingual, em $85 \%$ dos casos não está associado ao apinhamento ${ }^{(18)}$. A localização dos caninos não irrompido é de fundamental importância para o prognóstico e para o plano de tratamento. No caso clínico relatado, a coroa do canino impactado encontrava-se na posição vertical e sua inclinação estava por palatina, por isso, optou-se pelo tracionamento ortodôntico.

Após a manobra cirúrgica escolhida pelo profissional, inicia-se a fase ortodôntica de tração dos caninos que pode ser realizada com dispositivos fixos ou removíveis ${ }^{(19)}$. No caso relatado acima após a manobra cirúrgica e exposição da coroa do canino impactado, optamos pela tração do canino impactado através da colagem de gancho para o tracionamento ortodôntico, sendo utilizado como ancoragem um aparelho removível.

Tanto para o tracionamento ortodôntico como para correção da maloclusão, podemos utilizar alguns aparelhos fixos ou removíveis. Aparelho fixo metálico, aparelho fixo estético, aparelho autoligado, aparelho expansor palatino, aparelho removível e até mesmo os aparelhos extrabucais ${ }^{(20)}$.
No caso clínico, foi colado um gancho ortodôntico no dente impactado, realizando ativações através de um fio amarrilho para tracionar o canino impactado adaptado a uma placa de acrílico removível para a ancoragem. Para a correção da maloclusão (mordida cruzada anterior), utilizou-se o aparelho torno invertido superior, sendo um aparelho favorável para o tratamento de mordida cruzada, a fim de promover a expansão do arco superior devido a inclinação vestibular dos dentes, fazendo com que aumente a distância intercanina, corrigindo a mordida cruzada.

O tracionamento do dente deve ser feito o mais rápido, se possível após a cirurgia, ou no máximo entre a segunda e a terceira semana após a cirurgia, não podendo demorar mais que esse prazo. A força exercida para fazer o tracionamento é essencial, se o uso de força for errado pode causar na paciente anquilose e reabsorção cervical extrema (21). No caso clínico acima o tracionamento foi iniciado sete dias após a cirurgia de remoção do odontoma, sendo a força exercida por uma tração utilizando amarrilho e ganchos ortodônticos adaptado a uma placa de acrílico como ancoragem ${ }^{(22)}$.

Alguns autores realizaram um estudo retrospectivo para avaliar a taxa de sucesso do uso de mini placas, e obtiveram 98,6\% de sucesso deste tipo de ancoragem, demonstrando que as minis placas são altamente previsíveis e bem-sucedidas em movimentos ortodônticos complexos e que os casos de perdas do acessório se deram pela inflamação de tecidos moles devido à má higiene ${ }^{(23)}$. A barra Trans Palatina segundo Zeno et al. (24) (2019) consiste em um aparelho eficaz, de baixo custo, de fácil confecção, e capaz de inibir a transferências de forças ortodônticas para os dentes adjacentes.

Diversos autores são unânimes em afirmarem, que a correção da mordida cruzada anterior deve ser iniciada assim que o diagnóstico for estabelecido e quando o paciente apresentar maturidade suficiente para entender o problema, podendo colaborar em seu tratamento ${ }^{(25)}$. Em mordidas cruzadas funcional ou dentária podem ser utilizadas terapêuticas menos agressivas para os tecidos envolvidos, com aparelhos que realizam a expansão lenta da maxila, promovendo remodelação óssea ou compensações dentárias. Os descruzamentos de dentes tanto superior quanto inferior pode ser realizado através do uso de aparelhos removíveis com mola, assim como aparelhos fixos com bandas, ganchos e elásticos, podendo também utilizar placa expansora ${ }^{(26)}$. 


\section{CONCLUSÃO}

O tracionamento de um dente não irrompido é um dos procedimentos que gera muitas discussões entre os profissionais da área. Muitas das vezes é muito difícil decidir entre tracionar o dente impactado ou extrai-lo. Não existe um protocolo definitivo para o tratamento de dentes impactados. Deve ser baseado em um planejamento multidisciplinar, baseando-se em exames complementares, como exames radiográficos e exames clínicos.

É muito importante que o cirurgião dentista tenha consciência em saber quando mudar seu planejamento, o momento correto de mudar um planejamento inicial depois de visualizar os exames complementares e sempre atendendo às necessidades atuais dos pacientes devolvendo estética $\mathrm{e}$ sua função mastigatória.

\section{REFERÊNCIAS}

1. Karjodkar FR, Mali S, Sontakke S, Sansare K, Patil DJ. Five developmental anomalies in a single patient: a rare case report. J Clin Diagn Res. 2012; 6(9):1603-5.

2. Guttal KS, Naikmasur VG, Bhargava P, Bathi RJ. Frequency of developmental dental anomalies in the Indian population. Eur J Dent. 2010; $4(3): 263-9$

3. Almeida TE, Saa VJS, Kawakami PY, Palis CA, Mariani PB, Dottore AM Hiperdontia: relato de caso com 8 elementos supranumerários. Revista de Odontologia. Universidade Cidade de São Paulo. 2010; 22(1):78-84.

4. Nascimento FB, Parmagnani AA, Matheus RA, Stabile CLP, Furtado DR. Dente impactado associado a odontoma composto: relato de caso clínico. Rev. Uningá. 2019; 56(S7): 180-186.

5. Almeida GM, Cavalcanti RBMS, Medeiros GS, Almeida MDA, Castro RM, Andrade MA, Gomes LL, Moura ABR, Maia LS, Gomes ENS, Arruda LFA, Freitas GB, Ribeiro ED, Rocha JF. Use of included teeth: case reports. Research, Society and Development. 2020; 9(7): 1- 21

6. Soares-Santos KS, Albuquerque RB, Caldas LTS et al. Impactação bilateral de caninos superiores: relato de caso. Rev. ACBO. 2018; 27(1): 32-35.

7. Silva K, Santos DCL, Negrete D, Flaiban E, Bortolin R, Santos RL. Tracionamento de caninos inclusos: revisão de literatura. Rev. Odontol. Univ. Cid. São Paulo. 2019; 31(3): 71-81.

8. Longo BC, Kuhn L, Tomasin MFM, Tomasin Neto A, Grizza G, Souza MDB. Surgical-orthodontic therapy of compound odontoma in the anterior maxila Tratamento cirúrgico e ortodôntico de odontoma complexo em região anterior de maxila. J Bras Patol Med Lab. 2020; 56: 1-6.

9. Cé P, Prazeres C, Santos F E, Woltmann M. Odontoma complexo: relato de caso clínico atípico / Complex odontoma: atypical clinical case report. Rev Fac Odontol Univ Passo Fundo. 2009;14(1):56-60.

10. Abrahams JM, McClure SA. Pediatric Odontogenic Tumors. Oral Maxillofac Surg Clin North Am. 2016;28 (1):45-58

11. Almeida MAO, Quintão CCA, Capelli JJ. Ortodontia funda- mentos e aplicações clínicas. 1a ed. Rio de Janeiro: Guanabara Koogan;2008.

12. Siriwardena BSMS, Crane H, O’Neill N, et al. Odontogenic tumors and lesions treated in a single specialist oral and maxillofacial pathology unit in the United Kingdom in 1992-2016.. Oral Surg Oral Med Oral Pathol Oral Radiol. 2019; 127(2): 151-66.
13. Dalessandri D, Parrini S, Rubiano R, Gallone, D Migliorati, M. Migliorati, M Impacted and transmigrant mandibular canines incidence, aetiology, and treatment: a systematic review. European Journal of Orthodontics, 2016; 39 (2):161-169.

14. Silva, C.P.F. Análise do percurso eruptivo dos caninos permanentes em radiografias Panorâmicas Dissertação (mestrado em medicina dentária) Universidade Fernando Pessoa, Porto Portugal. 2019; 42.

15. Dalessandri, D, Parrini S, Rubiano R, Gallone D, Migliorati M. Impacted and transmigrant mandibular canines incidence, aetiology, and treatment: a systematic review. Eur. J. Orthod., Oxford, 2017; 39 (2): 161-19.

16. Kaczor, K.U. Impacted Teeth: An Interdisciplinary Perspective. Advances in Clinical and Experimental Medicine, 2016; 25 (3): 575-585.

17. Lu P, Chew MK. Orthodontic-surical management of na unusual dilacerated maxillary incisor. J Orthod Sci. 201; 7:24-8.

18. Cruvinel MOB, Valle CVM, Pinto RO, Siqueira GLC, Weise CM, ValleCorotti KM. Utilização de cantiléver para tracionamento de canino impactado. Ortodontia SPO. 2018; 51(1): 74-9.

19. Silva K, Santos Dcl, Negrete D, Flaiban E, Bortolin R, Santos R1 Tracionamento de caninos inclusos: revisão de literatura. Rev. Odontol. Univ. Cid. São Paulo 2019; 31(3): 71-81

20. Martteli, Karine et al. Caninos permanentes impactados por palatino: Uma alternativa de tratamento. Revista Uningá, [S,1]. 2012; 32 (1).

21. Proffit WR, Fields HW, Sarver D. Ortodontia contemporânea. Rio de Janeiro: Elsevier; 2007

22. Sankaran S, Kumar B, Prabhat MPV. Peripheral ossifying fibroma. Online J Health Allied Scs. 2009; 8 (3):1-3.

23. Lam R, Goonewardene SM, Allanc BP, Sugawara J. Success rates of a skeletal anchorage system in orthodontics: A retrospective analysis. Angle Orthod. 2018; 88: 2734

24. Zeno KG, El-Mohtar SJ, Mustapha S, Ghafari JG. Finite element analysis of stresses on adjacent teeth during the traction of palatally impacted canines. Angle Orthod. 2019;89 (3):41825.

25. Tavares, S.; Cspistrano, A.; Tavares, N.; Fonseca Junior, A. Sistema autoligável - tratamento de apinhamentos moderados e severos sem extrações. Ortho Sci., Orthod. Sci. Pract. 2015; 8 (31): 294-304

26. Maltagliati, LA. Tratamento ortodôntico com o sistema autoligável: quando a extração é necessária. Rev. Clín. Ortod. Dental Press, Maringá. 2015; 14 (1): $8-22$.

27. Rossi LB, Pizzol KED, Boeck EM, Lunardi N, Garbin AJI. Correção de mordida cruzada anterior funcional com a terapia de pistas diretas planas: relato de caso. FOL/UNIMEP 2012; 22(2):45-50. 